\title{
Current controversies in the initial post-surgical radioactive iodine therapy for thyroid cancer: a narrative review
}

\author{
Ralph Blumhardt', Ely A Wolin ${ }^{1,2}$, William T Phillips', Umber A Salman', \\ Ronald C Walker ${ }^{3,4}$, Brendan C Stack Jr $^{5}$ and Darlene Metter ${ }^{1}$ \\ ${ }^{1}$ University of Texas Health Science Center at San Antonio, San Antonio, Texas, USA \\ ${ }^{2}$ San Antonio Military Medical Center, San Antonio, Texas, USA \\ ${ }^{3}$ Tennessee Valley VA Healthcare System, Nashville, Tennessee, USA \\ ${ }^{4}$ Vanderbilt University Medical Center, Nashville, Tennessee, USA \\ ${ }^{5}$ UAMS Thyroid Center, University of Arkansas for Medical Sciences, Little Rock, Arkansas, USA
}

Correspondence should be addressed to E A Wolin Emails

ely.wolin@us.af.mil or lewolin@hotmail.com

\begin{abstract}
Differentiated thyroid cancer (DTC) is the most common endocrine malignancy and the fifth most common cancer in women. DTC therapy requires a multimodal approach, including surgery, which is beyond the scope of this paper. However, for over 50 years, the postoperative management of the DTC post-thyroidectomy patient has included radioactive iodine (RAI) ablation and/or therapy. Before 2000, a typical RAI post-operative dose recommendation was $100 \mathrm{mCi}$ for remnant ablation, $150 \mathrm{mCi}$ for locoregional nodal disease, and 175-200 mCi for distant metastases. Recent recommendations have been made to decrease the dose in order to limit the perceived adverse effects of RAI including salivary gland dysfunction and inducing secondary primary malignancies. A significant controversy has thus arisen regarding the use of RAI, particularly in the management of the low-risk DTC patient. This debate includes the definition of the low-risk patient, RAI dose selection, and whether or not RAI is needed in all patients. To allow the reader to form an opinion regarding post-operative RAl therapy in DTC, a literature review of the risks and benefits is presented.
\end{abstract}
Key Words
- thyroid cancer
- radioactive iodine therapy
${ }^{131}$ I
- thyroid cancer staging
- thyroid cancer resistance

Endocrine-Related Cancer (2014) 21, R473-R484

\section{Introduction}

The incidence of differentiated thyroid cancer (DTC) has been rising (Chen et al. 2009) with 60000 new cases expected in 2013 (Siegel et al. 2013). This increase is not due to an improved detection rate alone, as the increase involves not only small tumors identified by ultrasound, but also tumors of all sizes, and this upward trend was identified before the routine use of screening ultrasound (Chen et al. 2009, Di Cristofano 2013). At the same time, there is a recent trend toward being less aggressive with initial DTC surgery in order to limit surgical morbidity (Zetoune et al. 2010, Shan et al. 2012).

Radioactive iodine (RAI) has been used for the postsurgical treatment of DTC for over 50 years. RAI dose selection is generally based on patient risk factors most often referenced from the current American Thyroid Association (ATA) guidelines (see below; Cooper et al. 2009). The use of relatively large doses of RAI, i.e. $200 \mathrm{mCi}$ of RAI or higher, is generally accepted for the management

Published by Bioscientifica Ltd 
of high-risk patients. Controversy, however, stems from those patients designated as low risk. This dispute pertains to the use of low-dose, i.e. $30 \mathrm{mCi}$, vs high-dose, i.e. $100-150 \mathrm{mCi}$, RAI in the low-risk DTC patient.

The following is a literature review pertaining to the post-thyroidectomy low-risk DTC patient, and the use of low- vs high-dose RAI ablation. We propose that before low-dose RAI ablation is adopted as the standard of care, longer term survival studies need to demonstrate that lowdose RAI ablation is equivalent to high-dose RAI ablation in preventing recurrent DTC. Long-term recurrence-free survival (RFS) and overall survival (OS) per se should be the ultimate standards by which these two treatments are compared. Recent reports recommending RAI ablation in selective low- and intermediate-risk post-operative DTC patients will also be discussed (Ibrahimpasic et al. 2012, Rosario et al. 2012). Finally, a literature review of the risks of RAI therapy will be presented.

\section{Discussion}

\section{Purposes of post-surgical RAI therapy}

The three goals of post-surgical RAI therapy in patients with differentiated DTC are enumerated by Tuttle et al. (2010):

i) to ablate residual normal thyroid tissue that may facilitate surveillance.

ii) As 'adjuvant therapy' because of the potential tumoricidal effect on residual microscopic RAI-avid disease.

iii) To provide a post-treatment whole-body scan often revealing unknown local or distant metastases.

While these goals are important, the ultimate endpoint of post-surgical ablation is to minimize DTC recurrence and death, primarily by eliminating residual normal thyroid tissue or residual microscopic disease that could be a focus for future recurrence.

\section{Recent ATA guidelines (2009) to assess the risk of DTC recurrence}

Low-risk patient: i) no local or distant metastases; ii) all macroscopic tumor resected; iii) no locoregional tumor invasion; iv) tumor lacks aggressive histology (e.g. tall cell, insular, and columnar cell carcinoma) or vascular invasion; and v) no ${ }^{131}$ I uptake outside the thyroid bed on initial post-treatment whole-body RAI scan (RxWBS), if performed (Cooper et al. 2009).
Intermediate-risk patient: i) microscopic tumor invasion into the peri-thyroidal soft tissues at initial surgery; ii) cervical lymph node metastases or ${ }^{131}$ I uptake outside the thyroid bed on initial RxWBS; or iii) aggressive histology or vascular invasion.

High-risk patient: i) macroscopic tumor invasion; ii) incomplete tumor resection; iii) distant metastases; and possibly iv) thyroglobulinemia out of proportion to RxWBS findings.

\section{Long-term RFS and OS are required to determine the best therapy}

DTC recurrences may not present until 10-20 years postablation (Mazzaferri 1997). A long-term study (median follow-up 15.7 years) by Mazzaferri \& Jhiang (1994) demonstrated that DTC patients not treated with RAI exhibited a continually increasing death rate for 30 years (Fig. 1). Patients receiving early RAI ablation had superior survival.

Another study on 1599 RAI-treated thyroid cancer patients from MD Anderson Cancer Center with long-term follow-up (median, 11 years) demonstrated an $11 \%$ death rate from DTC. Early RAI therapy was the single most important factor predicting long-term survival (Samaan et al. 1992).

Delayed recurrence and late-stage progression of thyroid cancer are probably related to dormancy and then reactivation of thyroid cancer cells that have already metastasized at the time of initial treatment (Ringel 2011).

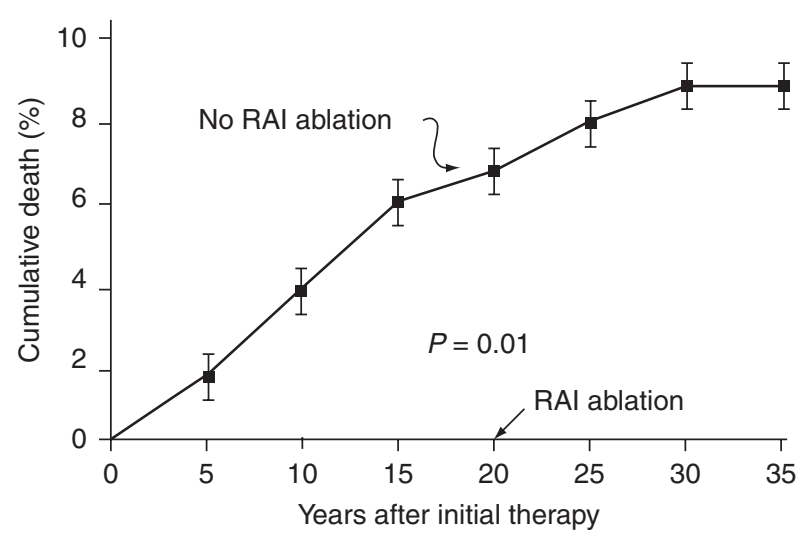

Figure 1

The relationship between thyroid cancer mortality and radioactive iodine ablation. Reprinted from Mazzaferri EL \& Jhiang SM 1994 Long-term impact of initial surgical and medical therapy on papillary and follicular thyroid cancer. American Journal of Medicine 97 418-428, with permission from Elsevier.

Published by Bioscientifica Ltd. 
Reactivation of metastatic DTC is hypothesized to be due to several factors, including changes in immune competence. Studies in patients with lymphoma have shown that cytotoxic CD8 T-lymphocytes are capable of maintaining tumors in a dormant state (Farrar et al. 1999). It is possible that reactivation of DTC metastases could be due to declining immune competence that is well known to occur with aging (Fulop et al. 2013). It is also possible that immune competency is significantly different between clinical populations. For example, diabetics with decreased immune competence have a significantly increased incidence of cancer (Starup-Linde et al. 2013). A recent study has also linked elevated BMI in childhood to an increased risk of developing DTC in adulthood (Kitahara et al. 2013). Similarly, the increased incidence of DTC in the USA correlates with the rising prevalence of obesity (Di Cristofano 2013, Pazaitou-Panayiotou et al. 2013). Populations with a high incidence of diabetes and obesity, such as those in South Texas (Duran-Gonzalez et al. 2011), have a higher rate of DTC compared with the general USA population (Lopez et al. 2013, Pappa \& Alevizaki 2013). Hence, long-term studies on RFS and OS in DTC patients treated with low- vs high-dose RAI early in their disease, carried out for at least 15 years, should not only consider clinical population diversity, but also address geographic differences.

\section{RAl dose controversy in initial treatment of the low-risk patient}

In 2012, two highly publicized New England Journal of Medicine articles (Mallick et al. 2012, Schlumberger et al. 2012) recommended $30 \mathrm{mCi}$ of RAI for all postthyroidectomy low-risk DTC patients. In the following review, the first (Schlumberger et al. 2012) will be referred to as Schlumberger's, and the second (Mallick et al. 2012) as Mallick's.

Both New England Journal of Medicine articles claim equivalent RAI ablation effectiveness with $30 \mathrm{vs} 100 \mathrm{mCi}$. However, the follow-up period of both studies was too short (6-9 months, Mallick and 8 months, Schlumberger) to determine whether the long-term RFS or OS was equivalent for both ablation doses. Mallick states, 'Our findings relate to thyroid ablation success at 6 to 8 months and do not address future recurrences' (Mallick et al. 2012). This statement is also true for Schlumberger's article. Nevertheless, Schlumberger states that ' $30 \mathrm{mCi}$ may be sufficient for the management of low-risk thyroid cancer' (Schlumberger et al. 2012). While the conclusions may be true over the short term, we contend that long-term RFS and OS could well be less with low-dose $(30 \mathrm{mCi})$ than with high-dose (e.g. $100 \mathrm{mCi}$ or more) RAI ablation performed early in the course of the disease.

\section{Concerns regarding Schlumberger's and Mallick's 2012 New England Journal of Medicine articles In} Schlumberger's article (Schlumberger et al. 2012), 27 out of 684 patients were excluded from randomization following therapy due to 'persistent disease' on RxWBS without mentioning which of these patients received $30 \mathrm{vs} 100 \mathrm{mCi}$ of RAI. It is likely that more of these patients were in the $100 \mathrm{mCi}$ group rather than the $30 \mathrm{mCi}$ group as higher doses are more likely to demonstrate an abnormal focus of activity on a post-RxWBS (Tuttle et al. 2010).

Schlumberger defines 'low-risk' DTC as pT1a (tumor diameter $<1 \mathrm{~cm}$ ) despite positive nodes, pT1b (tumor diameter $<2 \mathrm{~cm}$ ) despite positive nodes, and pT2 (tumor diameter $2-4 \mathrm{~cm}$ ) but only if there are no positive nodes. Schlumberger does not consider Nx (lymph nodes not evaluated by a pathologist) as appropriate in patients with pT2 lesions (Schlumberger et al. 2012). Why did these authors choose a $2 \mathrm{~cm}$ size cutoff? There are no data to suggest that less RAI is required to successfully treat a metastatic node with a primary tumor smaller than $2 \mathrm{~cm}$. Should any patient with nodal metastasis receive only $30 \mathrm{mCi}$ ? The National Comprehensive Cancer Network (NCCN) (2013) recommends initial treatment of nodal thyroid metastasis with at least $100 \mathrm{mCi}$ and up to $175 \mathrm{mCi}$ of RAI regardless of the primary tumor size (www.nccn.org/professionals/physician_gls/pdf/thyroid. pdf (free registration required), assessed 12/22/2013). This is concordant with the recommendation in UpToDate (9/18/13) of administering $150 \mathrm{mCi}$ of RAI for cervical and mediastinal node metastases (Tuttle 2012, www.uptodate. com/contents/differentiated-thyroid-cancer-radioiodinetreatment, assessed 12/22/2013).

In Mallick's article (Mallick et al. 2012), 21 patients (9.5\%) who received an initial $30 \mathrm{mCi}$ RAI dose underwent a second RAI treatment, when compared with nine patients $(4.1 \%)$ who received an initial $100 \mathrm{mCi}$ $(P=0.02)$. Out of these 30 retreated patients, one was retreated with $30 \mathrm{mCi}$, eight with $81-108 \mathrm{mCi}$, and 21 with $>108 \mathrm{mCi}$. Although Mallick concluded that $30 \mathrm{mCi}$ was an effective post-thyroidectomy RAI dose for DTC patients, the retreatment dose was $>30 \mathrm{mCi}$ in 29 out of 30 patients who failed initial RAI ablation. Clearly, the concern for successful ablation outweighed the concern for high-dose therapy risks.

Published by Bioscientifica Ltd. 
Schlumberger et al. cited articles do not fully support their conclusions Schlumberger et al., cited three publications (their references nos 4,5 , and 6) to support a claim of 1\% DTC recurrence rate after low-dose 'successful RAI ablation', which they define as i) a recombinant human thyrotropin (rhTSH)-stimulated thyroglobulin (Tg) level of $1.0 \mathrm{ng} / \mathrm{ml}$ or less and ii) a negative neck ultrasound at 6-12 months following RAI therapy. These citations are important and should be examined carefully as they provide the main supporting link for the contention that 'successful ablation' with $30 \mathrm{mCi}$ is as effective as high-dose RAI in preventing recurrences in low-risk patients.

In Schlumberger's cited article no. 4, Cailleux et al. (2000), 256 DTC patients were treated with $100 \mathrm{mCi}$ of RAI and followed up for 6 months- 9 years (median, 5 years). No patients received $30 \mathrm{mCi}$ of RAI. Thus, this article cannot support the position of a $1 \%$ or less DTC recurrence rate following $30 \mathrm{mCi}$ of RAI. With a median follow-up of 5 years, Cailleux et al. (2000) also did not address long-term follow-up.

Schlumberger's cited article no. 5, Pacini et al. (2002a), is a retrospective study carried out in 315 of 662 consecutive patients who received post-operative ablation with $30-100 \mathrm{mCi}$ of RAI and had a negative Tg at 6-12 months post-ablation. A diagnostic RAI WBS at that time was negative in 225 out of 315 (71.4\%) patients, while 90 (28.6\%) had residual thyroid bed activity; $61 / 90(67.7 \%)$ received a second ablation and $7 / 61$ (11.5\%) required two additional ablations, and $29 / 90(32.2 \%)$ patients were not retreated. Two patients $(0.6 \%)$ had recurrent disease. This article cannot support a $<1 \%$ recurrence rate with low-dose ablation. To do so would imply that the 61 retreated patients would have this low recurrence rate if they had not been retreated. It would also imply that there was no inherent bias for retreatment, including the seven patients who underwent not just one, but two additional RAI therapies. Furthermore, there was no discussion regarding which RAI dose was more effective as the subjects were treated over a wide range of $30-100 \mathrm{mCi}$, without knowing the individual ablation dose and follow-up.

In Schlumberger's cited article no. 6, Castagna et al. (2008), 85 DTC patients received $30-150 \mathrm{mCi}$ RAI, without any mention as to which dose was more effective. A wide range of RAI doses cannot be used to support the claim that successful ablation is associated with a $1 \%$ DTC recurrence rate in patients ablated with $30 \mathrm{mCi}$ of RAI. The clinical follow-up was from 13-228 months, median 48 months, and is also too short to demonstrate long-term RFS and OS recurrence rate as DTC recurrences occur up to 30 years after RAI ablation (Mazzaferri \& Jhiang 1994).

\section{Recent reviews of New England Journal of Medicine} articles by Schlumberger and Mallick These two New England Journal of Medicine 2012 articles were reviewed in the June 2013 Annals of Internal Medicine (Schlechte 2013) section entitled, 'Update in Endocrinology'. The critique mirrored our concern regarding insufficient long-term follow-up, stating 'The studies followed patients for only 6 to 9 months'. The review importantly concluded that 'Determining whether low risk patients can safely avoid the 100 mCi RAI dose will require another randomized study'. In other words, prospective, randomized, long-term studies comparing the efficacy of $30 \mathrm{vs} 100 \mathrm{mCi}$ RAI ablation in the low-risk DTC patient are required before we can conclude that ablation with $30 \mathrm{mCi}$ of $\mathrm{RAI}$ is as effective in preventing DTC recurrence.

Another review of the New England Journal of Medicine articles in the Journal of Endocrinology also agrees with the Annals of Internal Medicine review, stating that 'Ablation success is a surrogate endpoint - the ultimate endpoint is reduction in the number of recurrences or disease-related mortality' (Links \& van der Horst-Schrivers 2012).

Other studies of low- vs high-dose ablation In the peer-reviewed literature, not all studies report equivalent efficacy between 30 and $100 \mathrm{mCi}$ of RAI. A recent study on 259 DTC patients confined to the thyroid has reported that $100 \mathrm{mCi}$ of RAI was significantly more effective in thyroid ablation than $30-50 \mathrm{mCi}$ and also superior to $75 \mathrm{mCi}$ (Prpic et al. 2012). However, ablation rates were nearly similar after a second RAI dose. This second dose ranged from 30-100 mCi. Unfortunately, the authors did not explain their rationale for the second ablation dosage. Accordingly, this article cannot support low-dose ablation.

Three additional articles found low-dose RAI therapy to be less effective. In a meta-analysis of 19 studies, Doi \& Woodhouse (2000) compared 518 patients ablated with $30 \mathrm{mCi}$ against 449 ablated with $100 \mathrm{mCi}$, finding that $100 \mathrm{mCi}$ of RAI was significantly more effective and concluded that one in seven recurrences would have been prevented by high-dose ablation. In an expansion of their original report, Doi \& Woodhouse (2007) published a similar meta-analysis of risk of recurrence following high- vs low-dose ablation in 2584 patients (1094 low dose and 1490 high dose), finding further support for high-dose ablation in reduction of recurrent disease.

Published by Bioscientifica Ltd. 
Pacini et al. (2002b) stated that 'when using stimulation with rhTSH, a $30 \mathrm{mCi}$ standard dose of RAI is not sufficient for satisfactory thyroid ablation'.

\section{Limitations of classifying post-operative DTC patients as low-risk patients}

The following points address the clinical limitations in classifying post-thyroidectomy DTC patients as low risk:

i) as Mazzaferri states, 'cervical lymph node metastasis is associated with high recurrence rates' (Mazzaferri \& Jhiang 1994). This re-emphasizes the clinical postthyroidectomy dilemma, as there is no 'foolproof' method to confirm the presence, absence, or number of metastatic nodes or cells before RAI ablation. Determining whether there is any RAI activity outside the thyroid bed, one of the ATA guideline's criteria for separating low from intermediate risk is impossible without a diagnostic RAI WBS. In a 2010 article (Tuttle et al. 2010), 84 out of 394 (22\%) patients with DTC, with no known residual gross disease in the neck following surgery, demonstrated regional and/or distant metastasis on subsequent diagnostic or RxWBS examinations. Eight of the patients had distant metastasis. Apparently, initial locoregional metastases were found neither at the time of surgery, nor by the pathologist who analyzed the surgical specimen.

ii) Identifying metastatic nodes is dependent on the extent of nodal dissection and the intensity of pathological evaluation (Randolph et al. 2012). Even small central nodal metastases, which may only be detected with diligent histological evaluation, are clinically important. This has been demonstrated in a recent study reporting that nodal metastases $>2 \mathrm{~mm}$ in size had a sixfold greater risk of detectable Tg levels at 9 months after surgery (Lang et al. 2012).

iii) Can a low serum Tg value confirm that a DTC patient is a low-risk patient? Tuttle's group reported that a low non-stimulated serum $\mathrm{Tg}$ does not exclude RAI-avid metastatic foci in 46 out of 290 (16\%) intermediate-risk DTC patients (Robenshtok et al. 2013). They concluded, '...it is clear that RAI avid locoregional (and rare distant metastases) can be identified in intermediate and high-risk patients with non-stimulated or stimulated postoperative serum $\mathrm{Tg}$ levels $<0.4-0.6 \mathrm{ng} / \mathrm{ml}$ '. Although this study was in an intermediate-risk group, can we assume that their findings would not also be true in certain low-risk patients?

iv) How sensitive is neck ultrasound for detecting metastatic nodes? We have found no series addressing this question. Ultrasound is extremely operator dependent and does not delineate histology. Ultrasound only infers metastasis when there are sufficient malignant cells within the node to produce an anatomic change. Furthermore, Gallicchio et al. (2013) demonstrated lymph node involvement on post-therapy RAI SPECT/CT in 35\% of low-risk patients with micro-DTC and negative pre-therapy neck ultrasound. Such nodal disease clearly upstages the patient.

v) As with other malignancies, the primary DTC tumor size confers the risk of recurrence and death (Fig. 2; Mazzaferri \& Young 1981, Mazzaferri \& Jhiang 1995). Nonetheless, as Mazzaferri (2012) points out, '...small tumor size alone does not always assure low risk in incidentally identified thyroid cancers'.

A 35-year follow-up of tumors $<1 \mathrm{~cm}$ in size may identify regional and distant metastases, and relapses. Incidental tumors usually require little or no therapy,

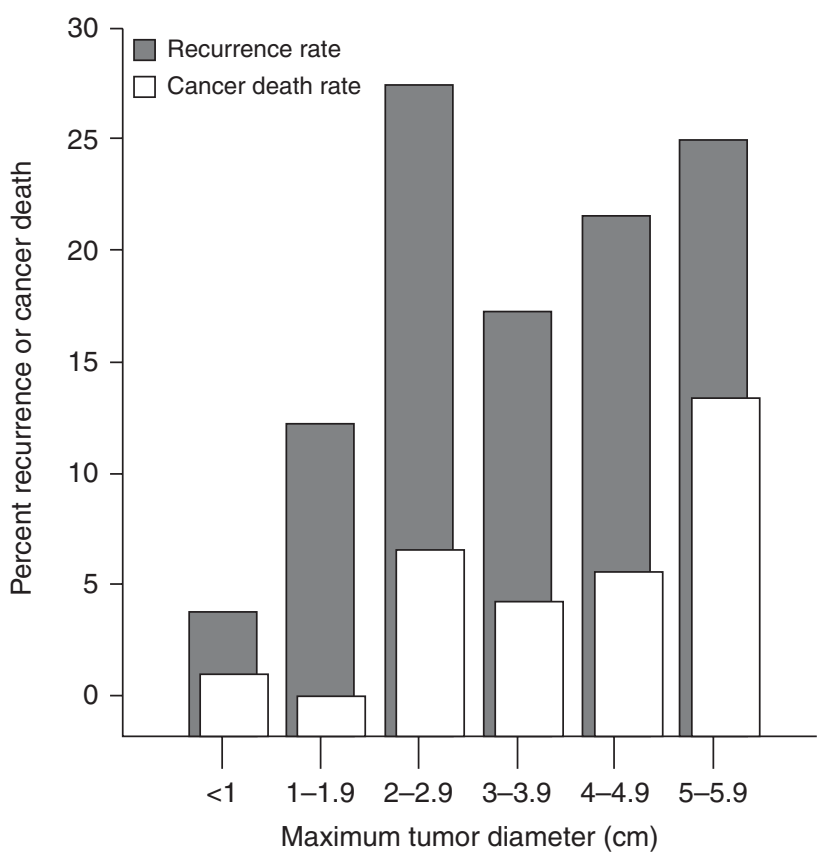

Figure 2

The relationship between thyroid cancer recurrence/mortality and tumor size. Reprinted from Mazzaferri EL \& Jhiang SM 1994 Long-term impact of initial surgical and medical therapy on papillary and follicular thyroid cancer. American Journal of Medicine 97 418-428, with permission from Elsevier.

Published by Bioscientifica Lto. 
whereas non-incidental thyroid tumors usually require vigorous treatment. In this case, 'incidental tumors' are thyroid cancers diagnosed in the final histopathology report after thyroid surgery for benign disease, such as thyroid nodules or hyperthyroidism (Lin et al. 2008).

In Mazzaferri's long-term study of DTC survival, his 'cut off' for low-risk disease was a primary tumor of size $<1.5 \mathrm{~cm}$ without lymph node metastasis (Mazzaferri \& Jhiang 1994). The New England Journal of Medicine articles define 'low risk' as a tumor $<2 \mathrm{~cm}$ in size with lymph node metastasis or a tumor $<4 \mathrm{~cm}$ in size without lymph node metastasis. Unfortunately, there are no long-term studies to support these different definitions of low risk.

Utilization of a preablation radioactive iodine scan by many nuclear medicine providers further outlines the limitations of clinical staging. For example, on preablation planar and SPECT/CT images after administration of $1 \mathrm{mCi}$ of ${ }^{131} \mathrm{I}$, Avram et al. (2013) identified unsuspected nodal metastases in 35\% (112 out of 320) of patients and unsuspected distant metastases in 7\% (23 out of 320) of patients. In this study, $57 \%$ of patients with a primary tumor $\leq 1 \mathrm{~cm}$ in size had nodal metastatic disease.

\section{The importance of ablation success and administration of RAl as soon as possible}

As with other cancers, it is probably best to treat DTC metastasis as early as possible. An investigation (Verburg et al. 2005) evaluating the prognostic significance of thyroid ablation reported an $87 \%$ 10-year disease-free survival after successful ablation with high-dose RAI and found that high-dose RAI had a one-third less risk of unsatisfactory ablation. The authors concluded that successful ablation 'as soon as possible' leads to a better prognosis and that the risks of high-dose RAI are insignificant compared with the estimated increase in life expectancy following successful ablation. Schlumberger et al. (1996) demonstrated that early treatment of lung metastasis significantly improves survival. Another group documented that patients with metastatic DTC who do not receive initial RAI treatment until more than 180 days after total thyroidectomy have a 4.2 times higher risk of death than those treated within 180 days (Higashi et al. 2011). Mazzaferri \& Jhiang (1994) also claimed that patients should be treated as early as possible. The patient adage 'do no harm', in DTC patients should include under-treatment by either low dose or delays in initial RAI therapy. In the end, we must keep in mind that most 'high-risk' patients began with 'low-risk' disease.

\section{Risks of RAI therapy}

Are those using $100 \mathrm{mCi}$ of RAI for post-operative DTC ablation causing more secondary cancers and significantly more permanent salivary gland problems than those using $30 \mathrm{mCi}$ of RAI? In recent years, concerns regarding the harmful effects of ionizing radiation from diagnostic imaging (typically X-rays from CT scanners) have been generalized to beta particle radiotherapy, i.e. RAI therapy. Importantly, Schlumberger's article stated that there was 'no difference in salivary gland problems' between those receiving $30 \mathrm{mCi}$ and those receiving $100 \mathrm{mCi}$ (Schlumberger et al. 2012). Lacrimal problems ('watery eyes') were also evaluated with little difference found between the ablation groups. If these perceived problems are related to the dose of administered activity, the number of salivary and lacrimal gland problems would be expected to be greater in the $100 \mathrm{mCi}$ group than in the $30 \mathrm{mCi}$ group.

\section{Salivary gland and dental risks from RAI therapy}

Mandel \& Mandel's article An article frequently referenced by those claiming a significant incidence of severe RAIinduced salivary gland damage by high-dose RAI is by Mandel \& Mandel (2003). Importantly, the article consists mostly of anecdotes. An image in this report from a sialogram demonstrates mild narrowing of Stensen's duct, presumed to be newly developed 17 months after RAI therapy. A neck CT image illustrates a mildly hyperdense parotid gland ' 10 months after RAI therapy'. This CT finding, however, is not diagnostic of significant permanent salivary gland damage. Colleagues of one of the authors described three patients with stomatitis, '...the origin of which can only be hypothesized'. However, 'a literature search revealed no such reports'. These authors suggest that these salivary gland side effects are dose dependent, occurring with doses $\geq 350 \mathrm{mCi}$.

This article does not provide sufficient objective evidence that high-dose RAI therapy produces significantly greater permanent salivary gland damage than low dose, nor does it discuss the incidence of these alleged salivary gland problems or other reported dysfunctions in a matched, untreated population, particularly when compared with an increased risk of recurrent DTC that may well occur with low-dose RAI at baseline.

Grewal et al.'s article Another series that reviewed salivary gland side effects in RAI-treated patients (Grewal et al. 2009) stated that ' ... when analysis was based on the entire treatment cohort (262 patients followed for a median of 7 years), persistent dry mouth was noted at the time of

Published by Bioscientifica Ltd. 
last follow-up in $2 \%$, altered taste in $1 \%$, salivary pain in $0.4 \%$, and tear duct blockage in $0.8 \%$. There was no significant difference in the administered RAI doses between those who did and those who did not develop dry mouth, alterations in taste, or salivary gland pain.

Quantifying salivary excretion To allow for an objective measure of salivary gland function following RAI therapy, several articles claim to quantify salivary excretion before and after lemon juice stimulation. However, there is no currently validated scintigraphic method for quantifying salivary gland function.

One major problem for this assessment is that there is no universal agreement on where to place the background region of interest (ROI). This seemingly 'minor' issue is critical in producing reliable results. If a mouth or oral background ROI is used, how can it be separated from salivary activity when the salivary glands are continually producing oral activity that is increasing background activity? Oral activity is also affected with swallowing. To address this problem, two groups recommend background ROIs outside the mouth. One used the brain (Bohuslavizki et al. 1996) and the other used the shoulder (Malpani et al. 1996). It is hard to understand as to how these remote sites could represent background activity in the salivary region. The second group of investigators (Malpani et al. 1996) also stated that the site of a background ROI must be placed with an accuracy of one pixel. This is not realistic.

${ }^{124}$ I PET/CT has also been recently investigated as a method to quantify the organ-specific absorbed dose to salivary glands from ${ }^{131} \mathrm{I}$ administration (Jentzen et al. 2010, Hobbs et al. 2013). Interestingly, the initial results have shown calculated radiation doses to the salivary glands that were less than expected. In these initial ${ }^{124} \mathrm{I}$ PET/CT dosimetry studies, the clinical effects of different doses have not been prospectively reviewed and a linear correlation between radiation dose and symptoms has not been reported. Future studies using ${ }^{124}$ I PET/CT, which clearly examine salivary gland radiation dosimetry and salivary function, will need to be carried out to determine whether currently administered ${ }^{131}$ I doses have radiationabsorbed dose ranges that are associated with significant salivary gland effects.

Despite the innate challenges in objectively assessing salivary gland function, there are a few articles attempting to show quantitative effects of RAI therapy on salivary gland function. A typical example is a recent paper (Jeong et al. 2013) attempting to measure differential salivary gland ejection fractions. This study reported a decrease in salivary gland function in 173 out of 852 patients (20\%) following RAI therapy. To our knowledge, salivary ejection fraction, as a technique, has never been validated and normal values regarding salivary gland ejection fractions have never been established. The authors did not describe what background ROI was used.

Another article (Bohuslavizki et al. 1996) cited by the same investigators used ${ }^{99 \mathrm{~m}}$ Tc-pertechnetate to measure salivary gland function before and after RAI therapy for both benign and malignant thyroid diseases. Their results indicated that even low RAI doses affected salivary gland function. Patients treated with $10.8-27 \mathrm{mCi}$ had mean post-treatment values that were equal to or lower than a group treated with $162-243 \mathrm{mCi}$. This reported finding is concerning as the lower doses fall into the therapy range for benign thyroid disease, and suggest that salivary gland side effects are not linearly related to RAI dose.

Accordingly, studies recommending an objective, absolute, and quantitative assessment of salivary function must be carefully scrutinized.

Risk of dental caries and oral problems A 2007 publication reported an increased incidence of dental caries following RAI therapy. The authors acknowledged that their results 'need independent confirmation' (Walter et al. 2007). The investigators were unable to link the increase in dental caries with sialadenitis or xerostomia. Their speculation for this apparent dichotomy was that (it) '....might be due to the fact that continuous variables, in contrast to dichotomous or categorical variables, facilitate the detection of significant predictors'. This is confusing. Nevertheless, the authors of UpToDate (9/18/13) (Tuttle 2012, www.uptodate.com/ contents/differentiated-thyroid-cancer-radioiodine-treatment, assessed 12/22/2013), referencing this article, stated that '...dental caries and teeth extractions were markedly higher in patients who developed post-RAI xerostomia'. The authors claimed that RAI might contribute to dental caries up to 15 years after administration, a surprising conclusion.

The same article cites the article by Mandel \& Mandel (2003) claiming that loss of taste, another potential complication of RAI, can become permanent. Mandel and Mandel did state that loss of taste may become permanent, but did not substantiate this conclusion with studies or references. Hence, this may be another example of a supposition becoming 'fact,' which can be misleading to unwary clinicians.

\section{Alleged increased risk of secondary primary malignancies from high-dose RAI therapy We} found only one large series (6871 patients) correlating

Published by Bioscientifica Ltd. 
RAI dose with secondary primary malignancies (SPMs) per number of person-years of follow-up (PYR) (Rubino et al. 2003). This study on 6871 patients is often cited. Although the unproven linear no-threshold model states that there is a linear relationship between an increasing radiation dose and a stochastic effect, referring to the incidence of SPMs, this article does not confirm this principle (Table 1).

These results are not linear. The article concluded that there was an excess of 53 cases of solid malignancy and three cases of leukemia per 10 years among 10000 patients treated with $100 \mathrm{mCi}$ of ${ }^{131} \mathrm{I}$. It appears that the assumed 'goal' of those recommended lower RAI doses is to further decrease these extremely low incidences of SPMs reported previously.

In a review and meta-analysis of two multicenter studies (one from Europe and the other from North America), Sawka et al. (2009) concluded that the risk of SPMs in thyroid cancer survivors treated with RAI is slightly increased compared with thyroid cancer survivors not treated with RAI.

Tuttle's group has recently published an analysis of the Surveillance, Epidemiology, and End Results (SEER) database and concluded that RAI therapy needed to be 'rationed' in low-risk DTC patients (Iyer et al. 2011). Tuttle reported an 'excess absolute risk' of 1.6 cases of leukaemia/10 000 PYR in RAI-treated patients. However, a significant limitation of this analysis is that the SEER program does not provide RAI administered doses; and hence is unable to correlate RAI dose with the risk of SPMs.

After analyzing the SEER database, another group came to a conclusion contrary to that of Tuttle's, stating that the use of RAI does not elevate the risk of SPMs. This group recommended that '...concern for SPM induction should not adversely affect the decision to administer RAI for DTC' (Bhattacharyya \& Chien 2006).

Table 1 Risk of SPM vs. cumulative RAI dose 2 or more years before SPM diagnosis

\begin{tabular}{|c|c|}
\hline RAl dose & SPM/PYR (\%) \\
\hline \multicolumn{2}{|c|}{ Solid cancers (mCi) } \\
\hline$<5.4$ & 0.6 \\
\hline $5.4-100$ & 0.9 \\
\hline $101-200$ & 0.5 \\
\hline $201-400$ & 0.7 \\
\hline$>400$ & 0.8 \\
\hline \multicolumn{2}{|c|}{ Leukemia (mCi) } \\
\hline$<5.4$ & 0.01 \\
\hline $5.4-100$ & 0.03 \\
\hline $101-500$ & 0.02 \\
\hline$>500$ & 0.00 \\
\hline
\end{tabular}

http://erc.endocrinology-journals.org DOI: 10.1530/ERC-14-0286
(C) 2014 Society for Endocrinology Printed in Great Britain
Verkooijen et al. (2006) reported an increased incidence of SPMs in DTC patients, but this increase was not related to RAI therapy. This brings to mind a 1968 article (Saenger et al. 1968) that reported an increased incidence of leukemia in patients treated with RAI for hyperthyroidism. The study also found that this increase in leukemia was similar in hyperthyroid patients treated with surgery. In other words, patients with thyroid disease had an increased incidence of leukemia, irrespective of whether or not they received RAI. A 1998 article from the same cooperative study (Ron et al. 1998) with a much longer follow-up of the same 35593 patients demonstrated no increase in overall cancer mortality in the RAI-treated hyperthyroid patients, with a standard mortality ratio of 1.02 . In 738831 person-years of follow-up, no increased overall cancer mortality was found in RAI-treated vs non-RAI-treated hyperthyroid patients. The authors also made the following important comment relevant to all RAI therapeutic interventions: '...the effectiveness of I-131 in killing thyroid cells presumably reduces the likelihood of malignant cell transformation (Ron et al. 1998)'.

\section{Recent trends recommending no post-operative RAI ablation in low- or intermediate-risk DTC patients}

A recent article proposes monitoring of post-thyroidectomy 'low-risk' DTC patients without RAI ablation (Rosario et al. 2012). Patients studied in this article had initial stimulated Tg values of $<1.0 \mathrm{ng} / \mathrm{ml}$, and their follow-up consisted of monitoring non-stimulated serum Tg levels and neck ultrasound. None of these patients had the further assurance of 'absent disease' with a negative post-RxWBS. Lymph node metastases were 'excluded' by ultrasound and the lack of suspicious nodes at surgery. Prophylactic cervical node dissection was not performed. Patient follow-up ranged from 12 to 72 months (mean, 44). However, the authors concluded that a larger number of patients with longer follow-up were needed to confirm this approach in the post-operative follow-up of 'low-risk' non-RAI-ablated DTC patients.

Another article (Ibrahimpasic et al. 2012) recommended no post-operative RAI ablation in lowand intermediate-risk patients with unstimulated Tg $<1.0 \mathrm{ng} / \mathrm{ml}$, claiming equivalent outcomes when comparing non-RAI-treated patients with RAI-treated patients. The authors support using unstimulated $\mathrm{Tg}$, '...because recurrence rates are so low with unstimulated $\mathrm{Tg}$ measurements, one can argue that the routine use of stimulated $\mathrm{Tg}$ in these patients to detect biochemical

Published by Bioscientifica Ltd 
disease without any anatomic demonstration has little practical value in clinical management'. Unfortunately, they do not clearly define 'anatomic demonstration'. Their follow-up consisted of an annual neck ultrasound and unstimulated Tg level. The limitations of this followup methodology for detecting DTC recurrence have been described earlier. The mean patient follow-up was a relatively short duration of 59 months (range 1-121 months). Careful scrutiny also reveals that this article's cohorts were not equivalent, and there were two major differences, which are as follows: i) out of 75 patients, 69 (92\%) with known nodal metastasis received RAI ablation, while $6(8 \%)$ were not ablated and ii) among the intermediate-risk patients, based on the authors' classification system, more than $90 \%(73 / 81)$ of patients were treated with RAI ablation while $<10 \%(8 / 81)$ were not. This indicates that the results are skewed against RAI ablation as the RAI-ablated cohort was at a higher risk than the cohort without ablation.

In addressing the practical clinical value of $\mathrm{Tg}$ surveillance, these authors state, 'It is important to note that our data cannot conclude that intermediate-risk patients who are young with large tumors and positive neck disease do not benefit from RAI'. Can we presume from this that the authors are certain that if these young patients have tumors $<4 \mathrm{~cm}$ in size, they would not benefit from RAI even though recurrences clearly do occur in patients with tumors $<4 \mathrm{~cm}$ in size (Fig. 2)? Moreover, how do the authors define 'positive neck disease', and how do they definitively exclude it?

An article by Tran Cao et al. (2012) addressed patient age and concluded, '...the presence of regional and metastatic thyroid cancer bears prognostic significance for all ages. Under current AJCC guidelines, young patients with metastatic thyroid cancer may be understaged'. Handkiewicz-Junak et al. (2007) evaluated 235 DTC patients aged 18 years or younger and reported that total thyroidectomy with modified lymphadenectomy performed for local metastasis, followed by RAI ablation, was associated with a substantial decrease in DTC locoregional recurrence, using a body-weight scaled high-dose protocol. Would this not also apply to DTC patients over the age of 18 ?

A recent publication has reported that the number of metastatic DTC nodes should be determined before post-operative RAI ablation is performed (Tuttle \& Sabra 2013). The article has recommended no RAI with nodal metastasis unless there are more than ten metastatic nodes that are $<5 \mathrm{~mm}$ in size, or more than five metastatic nodes of which the majority are $5-15 \mathrm{~mm}$ in size, or any single node more than $15 \mathrm{~mm}$ in size. The authors state, '...these are clearly arbitrary criteria for indications of RAI treatment' (emphasis ours; Tuttle \& Sabra 2013). Accordingly, the validity of this approach is unestablished, stressing the need for prospective trials that would confirm or refute these criteria and in order to firmly establish the clinical management that would minimize the risk of recurrent DTC. These authors' proposed arbitrary criteria defining significant node metastasis could under-stage up to $20 \%$ of DTC patients, as discussed above (Tuttle et al. 2010).

\section{Conclusion}

In our opinion, the current literature does not support either the routine use of $30 \mathrm{mCi}$ of RAI as an ablation dose or not ablating certain low- or intermediate-risk DTC patients. At all five of our institutions using a minimum dose of $100 \mathrm{mCi}$ for post-operative RAI therapy remains the standard of care, unless there are exceptional circumstances requiring dose reduction.

Studies comparing $30 \mathrm{mCi}$ with $100 \mathrm{mCi}$ RAI ablation have produced contradictory results, are mostly retrospective, and do not have sufficient long-term follow-up or adequately address cancer recurrence rates (Mallick et al. 2012). As a recent article from the Sloan-Kettering group points out, '....retrospective analysis can never take into account all the selection bias that is inherent in these studies (Ibrahimpasic et al. 2012)'. Furthermore, as discussed by Maxon et al. (1997), delivery of 300 Gy to remnant thyroid tissue, and $>80$ Gy to lymph node metastases, is required for an $80 \%$ success rate. In their report, not a single metastasis responded to therapy that received <35 Gy (Maxon et al. 1997). A $30 \mathrm{mCi}$ dose may be insufficient.

The significant limitations of the two recent New England Journal of Medicine articles recommending no postoperative RAI in low-risk patients are described above. In one article, the authors agree that their study should be repeated with a longer follow-up period (Rosario et al. 2012). In the other study, significant differences were present between the non-ablated and ablated cohorts (Ibrahimpasic et al. 2012). As discussed, the use of unstimulated Tg levels and neck ultrasound for followup is very concerning as both modalities have serious limitations.

Additionally, there is inadequate evidence in the peerreviewed literature to support the concept that high-dose RAI ablation causes harm compared with low-dose or even no RAI, regarding the incidence of SPMs or salivary gland

Published by Bioscientifica Ltd. 
dysfunction. To date, the evidence of serious risks associated with RAI therapy is weak and contradictory and, for the most part, is actually reassuring that any risk in RAI therapy is of a low magnitude or frequency. The potential risk of adverse RAI effects must be weighed against the risk of dying from recurrent DTC, which is $>8 \%$ in a 30-year follow-up period of patients not treated with RAI (Fig. 1). Long-term, e.g. 15 years, prospective randomized trials are needed to validate or negate the use of high, low, or no dose RAI ablation in the low- or intermediate-risk patient with DTC.

Therefore, the authors propose that until long-term studies support a change to low-dose ${ }^{131}$ I therapy, the low-risk DTC patient should continue to be initially treated as soon as possible with at least $100 \mathrm{mCi}$ of ${ }^{131} \mathrm{I}$, particularly given the trend toward less aggressive surgery that may result in leaving more thyroid tissue and/or tumors behind. Perhaps, a long-term prospective study utilizing preablation scanning with ${ }^{124}$ I PET dosimetry could permit a more exact calculation of an ${ }^{131} \mathrm{I}$ dose that will provide for successful ablation of remnant thyroid tissue and metastatic thyroid cancer using the lowest possible dose of ${ }^{131} \mathrm{I}$.

\section{Declaration of interest}

The authors declare that there is no conflict of interest that could be perceived as prejudicing the impartiality of this review.

\section{Funding}

This review did not receive any specific grant from any funding agency in the public, commercial or not-for-profit sector.

\section{References}

Avram AM, Fig LM, Frey KA, Gross MD \& Wong KK 2013 Preablation 131-I scans with SPECT/CT in postoperative thyroid cancer patients: what is the impact on staging? Journal of Clinical Endocrinology and Metabolism 98 1163-1171. (doi:10.1210/jc.2012-3630)

Bhattacharyya N \& Chien W 2006 Risk of second primary malignancy after radioactive iodine treatment for differentiated thyroid carcinoma. Annals of Otology, Rhinology, and Laryngology 115 607-610. (doi:10.1177/000348940611500806)

Bohuslavizki KH, Brenner W, Lassmann S, Tinnemeyer S, Tonshoff G, Sippel C, Wolf H, Clausen M \& Henze E 1996 Quantitative salivary gland scintigraphy in the diagnosis of parenchymal damage after treatment with radioiodine. Nuclear Medicine Communications 17 681-686. (doi:10.1097/00006231-199608000-00006)

Cailleux AF, Baudin E, Travagli JP, Ricard M \& Schlumberger M 2000 Is diagnostic iodine-131 scanning useful after total thyroid ablation for differentiated thyroid cancer? Journal of Clinical Endocrinology and Metabolism 85 175-178. (doi:10.1210/jcem.85.1.6310)

Castagna MG, Brilli L, Pilli T, Montanaro A, Cipri C, Fioravanti C, Sestini F, Capezzone M \& Pacini F 2008 Limited value of repeat recombinant human thyrotropin (rhTSH)-stimulated thyroglobulin testing in differentiated thyroid carcinoma patients with previous negative rhTSH-stimulated thyroglobulin and undetectable basal serum thyroglobulin levels. Journal of Clinical Endocrinology and Metabolism 93 76-81. (doi:10.1210/jc.2007-1404)

Chen AY, Jemal A \& Ward EM 2009 Increasing incidence of differentiated thyroid cancer in the United States, 1988-2005. Cancer 115 3801-3807. (doi:10.1002/cncr.24416)

Cooper DS, Doherty GM, Haugen BR, Kloos RT, Lee SL, Mandel SJ, Mazzaferri EL, McIver B, Pacini F, Schlumberger M et al. 2009 Revised American Thyroid Association management guidelines for patients with thyroid nodules and differentiated thyroid cancer. Thyroid 19 1167-1214. (doi:10.1089/thy.2009.0110)

Di Cristofano A 2013 Obesity and thyroid cancer: is leptin the (only) link? Endocrinology 154 2567-2569. (doi:10.1210/en.2013-1567)

Doi SA \& Woodhouse NJ 2000 Ablation of the thyroid remnant and ${ }^{131} \mathrm{I}$ dose in differentiated thyroid cancer. Clinical Endocrinology 52 765-773. (doi:10.1046/j.1365-2265.2000.01014.x)

Doi SA \& Woodhouse NJ 2007 Ablation of the thyroid remnant and ${ }^{131}$ I dose in differentiated thyroid cancer: a meta-analysis revisited2007. Clinical Medicine \& Research 5 87-90. (doi:10.3121/cmr.2007.763)

Duran-Gonzalez J, Ortiz I, Gonzales E, Ruiz N, Ortiz M, Gonzalez A, Sanchez EK, Curet E, Fisher-Hoch S, Rentfro A et al. 2011 Association study of candidate gene polymorphisms and obesity in a young Mexican-American population from South Texas. Archives of Medical Research 42 523-531. (doi:10.1016/j.arcmed.2011.10.010)

Farrar JD, Katz KH, Windsor J, Thrush G, Scheuermann RH, Uhr JW \& Street NE 1999 Cancer dormancy. VII. A regulatory role for CD8+ $\mathrm{T}$ cells and IFN- $\gamma$ in establishing and maintaining the tumor-dormant state. Journal of Immunology 162 2842-2849.

Fulop T, Larbi A \& Pawelec G 2013 Human T cell aging and the impact of persistent viral infections. Frontiers in Immunology 4 1-9. (doi:10.3389/ fimmu.2013.00271)

Gallicchio R, Giacomobono S, Capacchione D, Nardelli A, Barbato F, Nappi A, Pellegrino T \& Storto G 2013 Should patients with remnants from thyroid microcarcinoma really not be treated with iodine-131 ablation? Endocrine 44 426-433. (doi:10.1007/s12020-013-9935-9)

Grewal RK, Larson SM, Pentlow CE, Pentlow KS, Gonen M, Qualey R, Natbony L \& Tuttle RM 2009 Salivary gland side effects commonly develop several weeks after initial radioactive iodine ablation. Journal of Nuclear Medicine 50 1605-1610. (doi:10.2967/jnumed.108.061382)

Handkiewicz-Junak D, Wloch J, Roskosz J, Krajewska J, Kropinska A, Pomorski L, Kukulska A, Prokurat A, Wygoda Z \& Jarzab B 2007 Total thyroidectomy and adjuvant radioiodine treatment independently decrease locoregional recurrence risk in childhood and adolescent differentiated thyroid cancer. Journal of Nuclear Medicine 48 879-888. (doi:10.2967/jnumed.106.035535)

Higashi T, Nishii R, Yamada S, Nakamoto Y, Ishizu K, Kawase S, Togashi K, Itasaka S, Hiraoka M, Misaki T et al. 2011 Delayed initial radioactive iodine therapy resulted in poor survival in patients with metastatic differentiated thyroid carcinoma: a retrospective statistical analysis of 198 cases. Journal of Nuclear Medicine 52 683-689. (doi:10.2967/jnumed. 110.081059)

Hobbs RF, Jentzen W, Bockisch A \& Sgouros G 2013 Monte Carlo-based 3-dimensional dosimetry of salivary glands in radioiodine treatment of differentiated thyroid cancer estimated using ${ }^{124}$ I PET. Quarterly Journal of Nuclear Medicine and Molecular Imaging 57 79-91.

Ibrahimpasic T, Nixon IJ, Palmer FL, Whitcher MM, Tuttle RM, Shaha A, Patel SG, Shah JP \& Ganly I 2012 Undetectable thyroglobulin after total thyroidectomy in patients with low- and intermediate-risk papillary thyroid cancer - is there a need for radioactive iodine therapy? Surgery 152 1096-1105. (doi:10.1016/j.surg.2012.08.034)

Iyer NG, Morris LG, Tuttle RM, Shaha AR \& Ganly I 2011 Rising incidence of second cancers in patients with low-risk (T1N0) thyroid cancer who receive radioactive iodine therapy. Cancer $1174439-4446$. (doi:10.1002/cncr.26070) 
Jentzen W, Hobbs RF, Stahl A, Knust J, Sgouros G \& Bockisch A 2010 Pre-therapeutic ${ }^{124}$ I PET(/CT) dosimetry confirms low average absorbed doses per administered ${ }^{131}$ I activity to the salivary glands in radioiodine therapy of differentiated thyroid cancer. European Journal of Nuclear Medicine and Molecular Imaging 37 884-895. (doi:10.1007/s00259-009. 1351-2)

Jeong SY, Kim HW, Lee SW, Ahn BC \& Lee J 2013 Salivary gland function 5 years after radioactive iodine ablation in patients with differentiated thyroid cancer: direct comparison of pre- and postablation scintigraphies and their relation to xerostomia symptoms. Thyroid 23 609-616. (doi:10.1089/thy.2012.0106)

Kitahara CM, Gamborg M, Berrington de Gonzalez A, Sorensen TI \& Baker JL 2013 Childhood height and body mass index were associated with risk of adult thyroid cancer in a large cohort study. Cancer Research 74 1-8. (doi:10.1158/0008-5472.CAN-13-2228)

Lang BH, Tang AH, Wong KP, Shek TW, Wan KY \& Lo CY 2012 Significance of size of lymph node metastasis on postsurgical stimulated thyroglobulin levels after prophylactic unilateral central neck dissection in papillary thyroid carcinoma. Annals of Surgical Oncology 19 3472-3478. (doi:10.1245/s10434-012-2385-4)

Lin JD, Kuo SF, Chao TC \& Hsueh C 2008 Incidental and nonincidental papillary thyroid microcarcinoma. Annals of Surgical Oncology $\mathbf{1 5}$ 2287-2292. (doi:10.1245/s10434-008-9958-2)

Links TP \& van der Horst-Schrivers AN 2012 Thyroid cancer: successful remnant ablation - what is success? Nature Reviews. Endocrinology $\mathbf{8}$ 514-515. (doi:10.1038/nrendo.2012.113)

Lopez R, Agullo P \& Lakshmanaswamy R 2013 Links between obesity, diabetes and ethnic disparities in breast cancer among Hispanic populations. Obesity Reviews 14 679-691. (doi:10.1111/obr.12030)

Mallick U, Harmer C, Yap B, Wadsley J, Clarke S, Moss L, Nicol A, Clark PM, Farnell K, McCready R et al. 2012 Ablation with low-dose radioiodine and thyrotropin alfa in thyroid cancer. New England Journal of Medicine 366 1674-1685. (doi:10.1056/NEJMoa1109589)

Malpani BL, Samuel AM \& Ray S 1996 Quantification of salivary gland function in thyroid cancer patients treated with radioiodine. International Journal of Radiation Oncology, Biology, Physics 35 535-540. (doi:10.1016/S0360-3016(96)80016-2)

Mandel SJ \& Mandel L 2003 Radioactive iodine and the salivary glands. Thyroid 13 265-271. (doi:10.1089/105072503321582060)

Maxon HR, Thomas SR \& Samaratunga RC 1997 Dosimetrich considerations in the radioiodine treament of macrometastases and micrometasases from differentiated thyroid cancer. Thyroid 7 183-187. (doi:10.1089/ thy.1997.7.183)

Mazzaferri EL 1997 Thyroid remnant ${ }^{131}$ I ablation for papillary and follicular thyroid carcinoma. Thyroid 7 265-271. (doi:10.1089/thy. 1997.7.265)

Mazzaferri EL 2012 Managing thyroid microcarcinomas. Yonsei Medical Journal 53 1-14. (doi:10.3349/ymj.2012.53.1.1)

Mazzaferri EL \& Jhiang SM 1994 Long-term impact of initial surgical and medical therapy on papillary and follicular thyroid cancer. American Journal of Medicine 97 418-428. (doi:10.1016/0002-9343(94)90321-2)

Mazzaferri EL \& Jhiang SM 1995 Differentiated thyroid cancer long-term impact of initial therapy. Transactions of the American Clinical and Climatological Association 106 151-168 (discussion 168-170).

Mazzaferri EL \& Young RL 1981 Papillary thyroid carcinoma: a 10 year follow-up report of the impact of therapy in 576 patients. American Journal of Medicine 70 511-518. (doi:10.1016/0002-9343(81)90573-8)

National Comprehensive Cancer Network Clinical Practice Guidelines in Oncology for Thyroid Carcinoma, version 2.2013 (www.nccn.org/ professionals/physician_gls/pdf/thyroid.pdf (free registration required), assessed 12/22/2013).

Pacini F, Capezzone M, Elisei R, Ceccarelli C, Taddei D \& Pinchera A 2002a Diagnostic 131-iodine whole-body scan may be avoided in thyroid cancer patients who have undetectable stimulated serum Tg levels after initial treatment. Journal of Clinical Endocrinology and Metabolism $\mathbf{8 7}$ 1499-1501. (doi:10.1210/jcem.87.4.8274)
Pacini F, Molinaro E, Castagna MG, Lippi F, Ceccarelli C, Agate L, Elisei R \& Pinchera A $2002 b$ Ablation of thyroid residues with $30 \mathrm{mCi}$ (131)I: a comparison in thyroid cancer patients prepared with recombinant human TSH or thyroid hormone withdrawal. Journal of Clinical Endocrinology and Metabolism 87 4063-4068. (doi:10.1210/jc.2001011918)

Pappa T \& Alevizaki M 2014 Obesity and thyroid cancer: a clinical update. Thyroid 24 190-199. (doi:10.1089/thy.2013.0232)

Pazaitou-Panayiotou K, Polyzos SA \& Mantzoros CS 2013 Obesity and thyroid cancer: epidemiologic associations and underlying mechanisms. Obesity Reviews 14 1006-1022. (doi:10.1111/obr.12070)

Prpic M, Dabelic N, Stanicic J, Jukic T, Milosevic M \& Kusic Z 2012 Adjuvant thyroid remnant ablation in patients with differentiated thyroid carcinoma confined to the thyroid: a comparison of ablation success with different activities of radioiodine (I-131). Annals of Nuclear Medicine 26 744-751. (doi:10.1007/s12149-012-0637-9)

Randolph GW, Duh QY, Heller KS, LiVolsi VA, Mandel SJ, Steward DL, Tufano RP \& Tuttle RM 2012 The prognostic significance of nodal metastases from papillary thyroid carcinoma can be stratified based on the size and number of metastatic lymph nodes, as well as the presence of extranodal extension. Thyroid 22 1144-1152. (doi:10.1089/thy.2012. 0043)

Ringel MD 2011 Metastatic dormancy and progression in thyroid cancer: targeting cells in the metastatic frontier. Thyroid 21 487-492. (doi:10.1089/thy.2011.2121)

Robenshtok E, Grewal RK, Fish S, Sabra M \& Tuttle RM 2013 A low postoperative nonstimulated serum thyroglobulin level does not exclude the presence of radioactive iodine avid metastatic foci in intermediate-risk differentiated thyroid cancer patients. Thyroid $\mathbf{2 3}$ 436-442. (doi:10.1089/thy.2012.0352)

Ron E, Doody MM, Becker DV, Brill AB, Curtis RE, Goldman MB, Harris BS III, Hoffman DA, McConahey WM, Maxon HR et al. 1998 Cancer mortality following treatment for adult hyperthyroidism. Cooperative Thyrotoxicosis Therapy Follow-up Study Group. Journal of the American Medical Association 280 347-355. (doi:10.1001/jama.280.4.347)

Rosario PW, Mineiro Filho AF, Prates BS, Silva LC \& Calsolari MR 2012 Postoperative stimulated thyroglobulin of less than $1 \mathrm{ng} / \mathrm{ml}$ as a criterion to spare low-risk patients with papillary thyroid cancer from radioactive iodine ablation. Thyroid 22 1140-1143. (doi:10.1089/thy. 2012.0190)

Rubino C, de Vathaire F, Dottorini ME, Hall P, Schvartz C, Couette JE, Dondon MG, Abbas MT, Langlois C \& Schlumberger M 2003 Second primary malignancies in thyroid cancer patients. British Journal of Cancer 89 1638-1644. (doi:10.1038/sj.bjc.6601319)

Saenger EL, Thoma GE \& Tompkins EA 1968 Incidence of leukemia following treatment of hyperthyroidism. Preliminary report of the Cooperative Thyrotoxicosis Therapy Follow-Up Study. Journal of the American Medical Association 205 855-862. (doi:10.1001/jama.1968. 03140380059014)

Samaan NA, Schultz PN, Hickey RC, Goepfert H, Haynie TP, Johnston DA \& Ordonez NG 1992 The results of various modalities of treatment of well differentiated thyroid carcinomas: a retrospective review of 1599 patients. Journal of Clinical Endocrinology and Metabolism 75 714-720. (doi:10.1210/jcem.75.3.1517360)

Sawka AM, Thabane L, Parlea L, Ibrahim-Zada I, Tsang RW, Brierley JD, Straus S, Ezzat S \& Goldstein DP 2009 Second primary malignancy risk after radioactive iodine treatment for thyroid cancer: a systematic review and meta-analysis. Thyroid 19 451-457. (doi:10.1089/thy.2008.0392)

Schlechte JA 2013 Update in endocrinology: evidence published in 2012. Annals of Internal Medicine 158 821-824. (doi:10.7326/0003-4819-15811-201306040-00106)

Schlumberger M, Challeton C, De Vathaire F, Travagli JP, Gardet P, Lumbroso JD, Francese C, Fontaine F, Ricard M \& Parmentier C 1996 Radioactive iodine treatment and external radiotherapy for lung and bone metastases from thyroid carcinoma. Journal of Nuclear Medicine $\mathbf{3 7}$ 598-605. 
Schlumberger M, Catargi B, Borget I, Deandreis D, Zerdoud S, Bridji B, Bardet S, Leenhardt L, Bastie D, Schvartz C et al. 2012 Strategies of radioiodine ablation in patients with low-risk thyroid cancer. New England Journal of Medicine 366 1663-1673. (doi:10.1056/ NEJMoa1108586)

Shan C, Zhang W, Jiang D, Zheng X, Liu S \& Qiu M 2012 Routine central neck dissection in differentiated thyroid carcinoma: a systematic review and meta-analysis. Laryngoscope 122 797-804. (doi:10.1002/lary. 22162)

Siegel R, Naishadham D \& Jemal A 2013 Cancer statistics, 2013. CA: A Cancer Journal for Clinicians 63 11-30. (doi:10.3322/caac.21166)

Starup-Linde J, Karlstad O, Eriksen SA, Vestergaard P, Bronsveld H, Vries FD, Andersen M, Auvinen A, Haukka J, Hjellvik V et al. 2013 CARING (CAncer Risk and INsulin analoGues): the association of diabetes mellitus and cancer risk with focus on possible determinants - a systematic review and a meta-analysis. Current Drug Safety 8 296-332. (doi:10.2174/15748863113086660071)

Tran Cao HS, Johnston LE, Chang DC \& Bouvet M 2012 A critical analysis of the American Joint Committee on Cancer (AJCC) staging system for differentiated thyroid carcinoma in young patients on the basis of the Surveillance, Epidemiology, and End Results (SEER) registry. Surgery 152 145-151. (doi:10.1016/j.surg.2012.02.015)

Tuttle RM 2012 (www.uptodate.com/contents/differentiated-thyroidcancer-radioiodine-treatment, assessed 12/22/2013) Differentiated thyroid cancer: radioiodine treatment. In UpToDate. Ed DS Basow. Waltham, MA.
Tuttle RM \& Sabra MM 2013 Selective use of RAI for ablation and adjuvant therapy after total thyroidectomy for differentiated thyroid cancer: a practical approach to clinical decision making. Oral Oncology 49 676-683. (doi:10.1016/j.oraloncology.2013.03.444)

Tuttle RM, Lopez N, Leboeuf R, Minkowitz SM, Grewal R, Brokhin M, Omry G \& Larson S 2010 Radioactive iodine administered for thyroid remnant ablation following recombinant human thyroid stimulating hormone preparation also has an important adjuvant therapy function. Thyroid 20 257-263. (doi:10.1089/thy.2009.0401)

Verburg FA, de Keizer B, Lips CJ, Zelissen PM \& de Klerk JM 2005 Prognostic significance of successful ablation with radioiodine of differentiated thyroid cancer patients. European Journal of Endocrinology 152 33-37. (doi:10.1530/eje.1.01819)

Verkooijen RB, Smit JW, Romijn JA \& Stokkel MP 2006 The incidence of second primary tumors in thyroid cancer patients is increased, but not related to treatment of thyroid cancer. European Journal of Endocrinology 155 801-806. (doi:10.1530/eje.1.02300)

Walter MA, Turtschi CP, Schindler C, Minnig P, Muller-Brand J \& Muller B 2007 The dental safety profile of high-dose radioiodine therapy for thyroid cancer: long-term results of a longitudinal cohort study. Journal of Nuclear Medicine 48 1620-1625. (doi:10.2967/jnumed.107. 042192)

Zetoune T, Keutgen X, Buitrago D, Aldailami H, Shao H, Mazumdar M, Fahey T \& Zarnegar R 2010 Prophylactic central neck dissection and local recurrence in papillary thyroid cancer: a meta-analysis. Annals of Surgical Oncology 17 3287-3293. (doi:10.1245/s10434-010-1137-6)

Received in final form 22 September 2014

Accepted 2 October 2014

Made available online as an Accepted Preprint

2 October 2014
(C) 2014 Society for Endocrinology Printed in Great Britain 